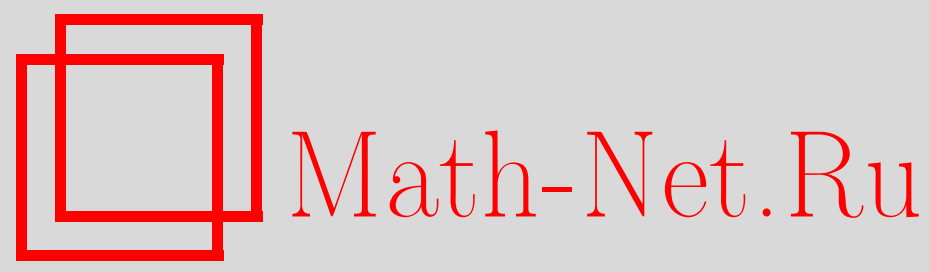

В. И. Буслаев, С. Ф. Буслаева, О теореме Пуанкаре о разностных уравнениях, Матем. заметки, 2005, том 78, выпуск $6,943-947$

DOI: https://doi.org/10.4213/mzm2664

Использование Общероссийского математического портала Math-Net.Ru подразумевает, что вы прочитали и согласны с пользовательским соглашением http://www . mathnet.ru/rus/agreement

Параметры загрузки:

IP : 54.224 .60 .19

26 апреля 2023 г., $14: 27: 44$ 


\section{О ТЕОРЕМЕ ПУАНКАРЕ О РАЗНОСТНЫХ УРАВНЕНИЯХ}

\section{В.И. Буслаев, С.Ф. Буслаева}

В данной статье показьвается, что функция, коэффициенты разложения которой в степенной ряд являются решением разностного уравнения с коэффициентами, стремящимися к пределам со скоростью геометрической прогрессии, допускает мероморфное продолжение за пределы круга сходимости.

К разностным уравнениям

$$
f_{n+k}+\alpha_{n, 1} f_{n+k-1}+\cdots+\alpha_{n, k} f_{n}=0, \quad n=0,1, \ldots,
$$

связывающим между собой элементы числовой последовательности $\left\{f_{n}\right\}_{n=1-k}^{\infty}$ приводят многие задачи анализа и теории чисел. Так, например, хорошо известно, что элементы последовательностей числителей $\left\{p_{n}\right\}_{n=0}^{\infty}$ и знаменателей $\left\{q_{n}\right\}_{n=0}^{\infty}$ числовой непрерывной дроби

$$
a_{0}+\frac{a_{1}}{b_{1}+\frac{a_{2}}{b_{2}+\cdots}}
$$

связаны между собой соотношениями

$$
p_{n}=b_{n} p_{n-1}+a_{n} p_{n-2}, \quad q_{n}=b_{n} q_{n-1}+a_{n} q_{n-2}, \quad n=1,2, \ldots,
$$

т.е. являются решениями одного и того же разностного уравнения

$$
f_{n}=b_{n} f_{n-1}+a_{n} f_{n-2}, \quad n=1,2, \ldots,
$$

но с разными начальными условиями $p_{-1}=1, p_{0}=a_{0}$ и $q_{-1}=0, q_{0}=1$.

Разностное уравнение (1) назьвается уравнением с постоянными коэффичиентами, если $\alpha_{n, i}=\alpha_{i}$ при всех $n=0,1, \ldots, i=1, \ldots, k$. Многочлен $z^{k}+\alpha_{1} z^{k-1}+\cdots+\alpha_{k}$ назьвается характеристическим многочленом разностного уравнения (1). Легко проверяется, что общее решение разностного уравнения (1) с постоянными коэффициентами записывается в виде

$$
f_{n}=\sum_{i=1}^{l} \sum_{j=1}^{r_{i}} C_{i, j} \lambda_{i}^{n} n^{j-1}, \quad n=0,1, \ldots
$$

где $\lambda_{1}, \ldots, \lambda_{l}$ - корни характеристического многочлена кратностей соответственно $r_{1}, \ldots, r_{l}$ $\left(\sum_{i=1}^{l} r_{i}=k\right), C_{i, j}$ - некоторые постоянные, однозначно определяемые по начальным условиям $f_{0}, \ldots, f_{k-1}$ конкретного решения.

Из равенства (4) следует, что для всякого решения $\left\{f_{n}\right\}_{n=0}^{\infty}$ разностного уравнения (1) с постоянными коэффициентами соответствующая ему функция

$$
f(z)=\sum_{n=0}^{\infty} f_{n} z^{n}
$$

является рациональной функцией с возможными полюсами в точках $\lambda_{1}^{-1}, \ldots, \lambda_{l}^{-1}$. При этом, если корни характеристического многочлена различны по модулю, то существует предел

$$
\lim _{n \rightarrow \infty} \frac{f_{n+1}}{f_{n}}
$$

Работа выполнена при поддержке Российского фонда фундаментальных исследований, грант № 05-01-01027, программы РАН "Современные проблемы теоретической математики”, программы "Ведущие научные школы", грант № HШ-1551.2003.1, и фонда INTAS, грант № 03-51-6637. 
и этот предел равен одному из корней характеристического многочлена.

Разностное уравнение (1) назьвается уравнением с предельно постоянными коэффициентами, если

$$
\lim _{n \rightarrow \infty} \alpha_{n, i}=\alpha_{i}
$$

Многочлен $z^{k}+\alpha_{1} z^{k-1}+\cdots+\alpha_{k}$ и в этом случае назьвается характеристическим многочленом разностного уравнения (1). Найти решения разностного уравнения (1) в случае предельно постоянных коэффициентов в явном виде не представляется возможным. Однако последнее из вышеприведенных утверждений имеет место и в этом значительно более сложном случае, что составляет содержание теоремы Пуанкаре [1] - одной из самых тонких теорем в теории линейных разностных уравнений.

Теорема (Пуанкаре). Пусть последовательность $\left\{f_{n}\right\}_{n=0}^{\infty}$ является решением разностного уравнения (1) с предельно постоянными коэффициентами, корни характеристического многочлена которого различны по модулю. Тогда либо $f_{n}=0$ при всех $n \geqslant n_{0}$, либо существует предел

$$
\lim _{n \rightarrow \infty} \frac{f_{n+1}}{f_{n}}
$$

и этот предел равен одному из корней характеристического многочлена.

Простые примеры показывают, что условия теоремы Пуанкаре нельзя ослабить, не нарушив утверждающей части теоремы, а утверждающую часть нельзя усилить, не потребовав дополнительных предположений.

Имеется целый ряд результатов, в том или ином направлении уточняющих теорему Пуанкаpe. Одно из важнейших уточнений было получено Перроном [2] для невыро жденных разностных уравнений, т.е. для уравнений с дополнительным предположением $\alpha_{n, k} \neq 0, n=0,1, \ldots$ (невырожденность уравнения (1) означает возможность его решения в "другую сторону", т.е. возможность однозначного определения значения $f_{n}$ при известных значениях $f_{n+1}, \ldots, f_{n+k}$ ).

Теорема (Перрон). Пусть корни характеристического многочлена невырожденного разностного уравнения (1) с предельно постоянными коэффичиентами различны по модулю. Тогда для всякого корня $\lambda$ характеристического многочлена найдется решение $\left\{f_{n}\right\}_{n=0}^{\infty}$ разностного уравнения (1) такое, что

$$
\lim _{n \rightarrow \infty} \frac{f_{n+1}}{f_{n}}=\lambda \text {. }
$$

Так как множество решений разностного уравнения (1) образует $k$-мерное векторное пространство, то из теоремы Перрона следует, что всякое решение $\left\{f_{n}\right\}_{n=1-k}^{\infty}$ разностного уравнения (1) может быть записано в виде $f_{n}=C_{1} f_{n, 1}+\cdots+C_{k} f_{n, k}$, где $C_{1}, \ldots, C_{k}$ - некоторые постоянные числа,

$$
\lim _{n \rightarrow \infty} \frac{f_{n+1, i}}{f_{n, i}}=\lambda_{i}, \quad i=1, \ldots, k,
$$

$\lambda_{1}, \ldots, \lambda_{k}-$ корни характеристического многочлена. Если эти корни перенумеровать в порядке возрастания их модулей $\left|\lambda_{1}\right|<\cdots<\left|\lambda_{k}\right|$, то, как легко видеть,

и

$$
\lim _{n \rightarrow \infty}\left|f_{n, 1}\right|^{1 / n}=\left|\lambda_{1}\right|
$$

$$
\lim _{n \rightarrow \infty}\left|f_{n}\right|^{1 / n} \geqslant\left|\lambda_{2}\right|>\left|\lambda_{1}\right|
$$

для всякого решения $\left\{f_{n}\right\}_{n=1-k}^{\infty}$, отличного от $\left\{C f_{n, 1}\right\}_{n=1-k}^{\infty}$. Хорошо известно, что существование единственного (с точностью до постоянного множителя) решения минимального роста разностного уравнения (3) эквивалентно сходимости непрерьвной дроби (2). Поэтому как простое следствие теоремы Перрона может быть получена одна из самых красивых теорем теории непрерывных дробей - теорема Ван Флека [3] о сходимости правильной $C$-дроби

$$
a_{0}+\frac{a_{1} z}{1+\frac{a_{2} z}{1+\cdots}}
$$

с предельно постоянными коэффициентами. 
Теорема (Ван Флек). Пусть коэффичиенты правильной $C$-дроби (5) имеют предел

$$
\lim _{n \rightarrow \infty} a_{n}=a \neq 0 .
$$

Тогда $C$-дробь (5) сходится к мероморфной функции равномерно на компактах, лехсащих $\boldsymbol{\theta} \mathbb{C} \backslash \Gamma$, әде

$$
\Gamma=\left\{z \in \mathbb{C}: z=\frac{t}{4 a}, t \leqslant-1\right\},
$$

и не содержащих полюсов предельной функиии.

Заметим, что множество $\Gamma$ в теореме Ван Флека - это в точности множество тех $z \in \mathbb{C}$, при которых равны по модулю корни

$$
\lambda_{1,2}(z)=\frac{1 \pm \sqrt{1+4 a z}}{2}
$$

характеристического многочлена разностного уравнения

$$
f_{n}(z)=f_{n-1}(z)+a_{n} z f_{n-2}(z), \quad n=1,2, \ldots,
$$

соответствующего непрерьвной дроби (5).

Из других уточнений теоремы Пуанкаре отметим следующий резултат одного из соавторов [4].

ТЕОРема. Пусть әлементы последовательности $\left\{f_{n}\right\}_{n=0}^{\infty}$ удовлетворяют рекуррентным соотношениям

$$
f_{n}+\alpha_{n, 1} f_{n-1}+\cdots+\alpha_{n, n} f_{0}=0, \quad n=0,1, \ldots,
$$

бесконечного порядка с коэффичиентами $\alpha_{n, 1}, \ldots, \alpha_{n, n}$ такими, что многочлень $1+$ $\alpha_{n, 1} z+\cdots+\alpha_{n, n} z^{n}$ равномерно на компактах, лежащих в круге $\{|z|<R\}$, сходятся при $n \rightarrow \infty \kappa$ функиии $\alpha(z)$, все корни которой различны по модулю. Тогда либо

$$
\varlimsup_{n \rightarrow \infty}\left|f_{n}\right|^{1 / n} \leqslant \frac{1}{R}
$$

либо существует предел

$$
\lim _{n \rightarrow \infty} \frac{f_{n}}{f_{n+1}}
$$

u этот предел равен одному из корней функции $\alpha$.

В данной заметке теорема Пуанкаре уточняется на случай разностных уравнений с коэффициентами, сходящимися со скоростью геометрической прогрессии

$$
\varlimsup_{n \rightarrow \infty}\left|\alpha_{n, i}-\alpha_{i}\right|^{1 / n} \leqslant q<1, \quad i=1, \ldots, k .
$$

Этот случай занимает промежуточное место между общим случаем разностных уравнений с предельно постоянньми коэффициентами и частным случаем постоянных коэффициентов. Если в случае постоянных коэффициентов функция

$$
f(z)=\sum_{n=0}^{\infty} f_{n} z^{n},
$$

как отмечалось, является рациональной функцией с возможньми полюсами в точках $\lambda_{1}^{-1}, \ldots, \lambda_{k}^{-1}$, а в общем случае предельно постоянных коэффициентов можно утверждать только лишг, что радиус круга голоморфности функции $f$ равен одному из чисел $\left|\lambda_{1}^{-1}\right|, \ldots,\left|\lambda_{k}^{-1}\right|$, то в рассматриваемом промежуточном случае можно утверждать, что функция $f$ допускает мероморфное продолжение за пределы ее круга голоморфности. Более точно, в данной заметке будет доказана следующая теорема. 
Теорема 1. Пусть последовательность $\left\{f_{n}\right\}_{n=0}^{\infty}$ является решением разностного уравнения (1), коэффициенты которого удовлетворяют условию (6). Тогда

1) $\varlimsup_{n \rightarrow \infty}\left|f_{n}\right|^{1 / n}$ равен модулю одного из корней характеристического многочлена $\alpha(z)=z^{k}+\alpha_{1} z^{k-1}+\cdots+\alpha_{k} ;$

2) функчия $f(z)=\sum_{n=0}^{\infty} f_{n} z^{n}$ допускает мероморфное продолжение из круга

$$
\left\{|z|<\left(\varlimsup_{n \rightarrow \infty}\left|f_{n}\right|^{1 / n}\right)^{-1}\right\}
$$

голоморфности в круг

$$
\left\{|z|<q^{-1}\left(\varlimsup_{n \rightarrow \infty}\left|f_{n}\right|^{1 / n}\right)^{-1}\right\}
$$

и все полюсы функции $f$ в этом круге лежат среди нулей многочлена $z^{k} \alpha(1 / z)$; 3) если

$$
\varlimsup_{n \rightarrow \infty}\left|f_{n}\right|^{1 / n}>0
$$

то существует натуральное число $l$ такое, что последовательность $\left\{f_{n}\right\}_{n=0}^{\infty}$ удовлетворяет системе l соотношений

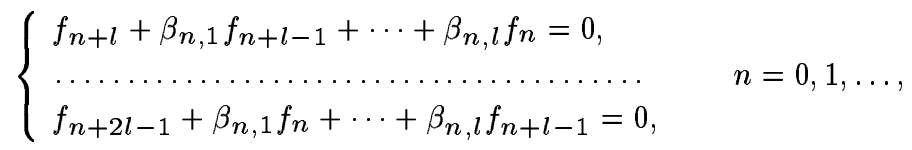

с предельно постоянными коэффициентами такими, что

$$
\varlimsup_{n \rightarrow \infty}\left|\beta_{n, i}-\beta_{i}\right|^{1 / n}<1, \quad i=1, \ldots, l,
$$

имногочлен $\beta(z)=z^{l}+\beta_{1} z^{l-1}+\cdots+\beta_{l}$ делит характеристический многочлен $\alpha(z)$.

ДоКАЗАТЕЛЬСтво. Утверждение 1) теоремы о верхнем пределе

$$
\varlimsup_{n \rightarrow \infty}\left|f_{n}\right|^{1 / n}
$$

доказано в работе [4] и имеет место в общем случае предельно постоянных коэффициентов без предположений (6).

Далее, можно предполагать, что $R_{0}(f)<\infty$, так как иначе доказьвать больше нечего. Из равенств (1) и (6) следует, что для любых фиксированных $\nu \in \mathbb{Z}$ и $\varepsilon>0$ при всех достаточно больших $n \geqslant n(\nu, \varepsilon)$ имеют место неравенства

$$
\begin{aligned}
& \left|f_{n+\nu+k}+\alpha_{1} f_{n+\nu+k-1}+\cdots+\alpha_{k} f_{n+\nu}\right| \\
& \quad=\mid\left(f_{n+\nu+k}+\alpha_{1} f_{n+\nu+k-1}+\cdots+\alpha_{k} f_{n+\nu}\right) \\
& \quad-\left(f_{n+\nu+k}+\alpha_{n+\nu, 1} f_{n+\nu+k-1}+\cdots+\alpha_{n+\nu, k} f_{n+\nu}\right) \mid \\
& \quad=\left|\left(\alpha_{1}-\alpha_{n+\nu, 1}\right) f_{n+\nu+k-1}+\cdots+\left(\alpha_{k}-\alpha_{n+\nu, k}\right) f_{n+\nu}\right| \leqslant(q+\varepsilon)^{n}\left(\varlimsup_{n \rightarrow \infty}\left|f_{n}\right|^{1 / n}\right)^{n} .
\end{aligned}
$$

Положим

$$
R_{0}(f)=\left(\varlimsup_{n \rightarrow \infty}\left|f_{n}\right|^{1 / n}\right)^{-1}
$$


и обозначим через $R_{m}(f)$ радиус максимального круга с центром в точке $z=0$, в который функция $f$, голоморфная в круге $\left\{|z|<R_{0}(f)\right\}$, мероморфно продолжается и имеет не более $m$ полюсов. Пусть $m \in \mathbb{Z}$ таково, что $R_{m-1}(f)<q^{-1} R_{0}(f)$. Тогда найдутся положительные числа $\varepsilon$ и $\delta$ такие, что $R_{m-1}(f)^{-1} e^{-\delta}>(q+\varepsilon) R_{0}(f)^{-1}$. При этих $\varepsilon$ и $\delta$ из неравенств (9) следуют равенства

$$
\left|f_{n+\nu+k}+\alpha_{1} f_{n+\nu+k-1}+\cdots+\alpha_{k} f_{n+\nu}\right|=o\left(R_{m-1}(f)^{-n} e^{-\delta n}\right), \quad n \rightarrow \infty, \quad \nu \in \mathbb{Z} .
$$

Полученные равенства справедливы при всех $\nu \in \mathbb{Z}$ и, в частности, при $\nu=0, \ldots, m-1$. В работе [4] показано, что из системы $m$ равенств $(10)$ (при $\nu=0, \ldots, m-1$ ) следует, что функция $f$ имеет $m$ особых точек (лежаших внутри или на границе круга $\left\{|z|<R_{m-1}(f)\right\}$ ), каждая из которых является корнем многочлена $\alpha$. Поэтому $m \leqslant \operatorname{deg} \alpha=k$. Это означает, что при $m>k$ неравенство $R_{m-1}(f)<q^{-1} R_{0}(f)$ невозможно. В частности, это равенство невозможно при $m=k+1$. Следовательно, $R_{k}(f) \geqslant q^{-1} R_{0}(f)$, т.е. функция $f$ допускает мероморфное продолжение в круг $\left\{|z|<q^{-1} R_{0}(f)\right\}$.

Покажем теперь, что все полюсы функции $f$ в круге $\left\{|z|<q^{-1} R_{0}(f)\right\}$ лежат среди корней многочлена $\alpha$. Рассмотрим минимальное из чисел $l=1, \ldots, k$, для которых $R_{l} \geqslant q^{-1} R_{0}(f)$, а $R_{l-1}(f)<q^{-1} R_{0}(f)$. По уже доказанному функция $f$ имеет $l$ особых точек, лежащих внутри или на границе круга $\left\{|z|<R_{l-1}(f)\right\}$, каждая из которых является корнем многочлена $\alpha$. Так как $R_{l-1}(f)<R_{l}(f)$, то каждая из этих особых точек является полюсом и других полюсов функция $f$ в круге $\left\{|z|<R_{l}(f)\right\}$, содержашем круг $\left\{|z|<q^{-1} R_{0}(f)\right\}$, не имеет.

Кроме того, так как функция $f$ имеет в круге $\left\{|z|<R_{l}(f)\right\}$ ровно $l$ полюсов, лежаших среди нулей многочлена $z^{k} \alpha(1 / z)$, то можно воспользоваться теоремой Монтессу де Болора [5] и получить как ее следствие утверждение 3 ) теоремы.

Отметим, что при помощи теоремы Гончара [6] неравенства (8) могут быть записаны в уточненном виде

$$
\varlimsup_{n \rightarrow \infty}\left|\lambda_{n, i}-\lambda_{i}\right|^{1 / n} \leqslant\left|\frac{\lambda_{i}}{R_{m}(f)}\right|<1, \quad i=1, \ldots, m,
$$

где $\lambda_{n, 1}, \ldots, \lambda_{n, m}$ - корни многочлена $z^{m}+\beta_{n, 1} z^{m-1}+\cdots+\beta_{n, m}$.

\section{СПИСОК ЦИТИРОВАННОЙ ЛИТЕРАТУРЫ}

1. Poincaré H. // Amer. J. Math. 1885. V. 7. P. 203-258. 2. Перрон O. // J. Reine Angew. Math. 1909. V. 136. P. 17-37; 1910. V. 137. P. 6-64. 3. Van Vleck E. V.// Trans. Amer. Math. Soc. 1904. V. 5. Р. 253-262. 4. Буслаев В. И. // Матем. сб. 1986. Т. 131 (173). №3 (11). С. 357-384. 5. de Montessus de Ballore R. // Bull. Soc. Math. France. 1902. V. 30. P. 28-36. 6. Гончар А. А. // Матем. сб. 1981. Т. 115(157). № 4. С. 590-613. 